\title{
Breve revisión histórica del autismo
}

RESUMEN: Reflexión acerca del desarrollo histórico y estado actual en la investigación del autismo.

PALABRAS CLAVES: Autismo, psicosis precoz, trastornos generalizados del desarrollo.
SUMMARY: Reflection on the historical development and present state of research into autism.

KEY WORDS: Autism, praecox psychosis, pervasive development disorders.

\section{Los inicios de la investigación del autismo}

Antes de abordar el trabajo pionero desarrollado por Leo Kanner (18941981) en el estudio del autismo, conviene recordar la elección desafortunada que dicho término supuso, dada la confusión que generó con el del autismo que en 1911 había usado Eugen Bleuer (1857-1939) para expresar la retirada activa con que algunos pacientes esquizofrénicos se repliegan al terreno de la fantasía, pues, a diferencia de Bleuer, que con él hacía referencia a la conducta intencional de evitar relaciones sociales, a lo que Kanner aludía era a la incapacidad para establecer tales vínculos relacionales. Asimismo, mientras en sentido bleueriano tal repliegue estaba conectado a la rica vida fantástica del sujeto esquizofrénico, eso no sucede en el autista, quien carece de tal ingrediente cognitivo. De ello algunos investigadores del autismo infirieron un posible nexo entre éste y la posterior esquizofrenia en la edad adulta, lo que influyó en la tendencia entre los psiquiatras infantiles de utilizar de forma intercambiable las etiquetas diagnósticas de esquizofrenia infantil, autismo y psicosis infantil (1).

En un intento de aislar el síndrome autista de las otras condiciones psiquiátricas mencionadas, fue necesario determinar qué síntomas eran universales y cuáles eran específicos, incluyéndose dentro de los primeros una incapacidad profunda y general para establecer relaciones con otros; retraso en la adquisición del lenguaje, que dificultaba la comprensión; ecolalia e inversión pronominal y fenómenos ritualistas o compulsivos, tales como la insistencia en la identidad, a la que Rivière (2) prefiere llamar trastorno de la función ejecutiva, al juzgar tal etiqueta más precisa y sustentada por nutrida investigación. La falta de respuesta social y las dificultades de lenguaje sólo pueden considerarse indicadores claros de autismo si no corresponden a la edad mental del niño y se explican exclusivamente como rasgos distintivos del trastorno autista ausentes en el desarrollo evolutivo normal. Dentro de los segundos, por el contrario, pueden evidenciarse movimientos repetitivos estereotipados (especialmente manierismos en las manos y en los dedos), poca capacidad de atención, retraso en el control de los esfínteres y conductas autolesivas. Además de confirmar los criterios diagnósticos formulados inicialmente por Kanner, 
a los que a continuación se harán referencia, con ello se logró una definición operativa del autismo en los dos grupos de síntomas antes referidos.

En efecto, partiendo de una muestra compuesta de 11 niños que manifestaban conductas extrañas no recogidas previamente en ningún sistema nosológico, Kanner (3) describió por vez primera el autismo como un síndrome comportamental cuyos rasgos distintivos eran alteraciones del lenguaje (o comunicación), de las relaciones sociales (a las que juzgó como el trastorno fundamental patognomónico del síndrome) y de los procesos cognitivos, dentro de los cuales algunos han resaltado los trastornos de la flexibilidad, cuya gravedad dependerá del nivel cognitivo, esto es, del nivel de profundidad del espectro autista $(4 ; 2)$. Previo a la formulación de tal diagnóstico y sus posibles intervenciones, Kanner (5) había ya expresado la necesidad de elaborar un detallado informe acerca del entorno familiar y social en que el niño vive, valiosísima información hoy eclipsada en los estudios recientes de autismo (6).

Con todo, esto último no impidió que Kanner (3) atribuyera como origen de tal incapacidad para establecer vínculos afectivos adecuados deficiencias innatas, de origen biológico, que paradójicamente no investigó (7), al concentrar sus esfuerzos en estudiar los rasgos de personalidad y tipo de interacción que los padres mantenían con sus hijos, abocando con ello a que en las dos décadas posteriores el grueso de las investigaciones conceptuaran el autismo como un desorden fundamentalmente de carácter emocional, lo que explicaría la mayor eclosión de trabajos de orientación psicoanalítica publicados acerca del autismo durante esos años respecto a los de otros modelos psicoterapéuticos (8).

De tales escritos psicoanalíticos pueden al menos mencionarse dos: La fortaleza vacía. Autismo infantil y el nacimiento del yo (9) y Una comprensión psicoanalítica del autismo infantil y su tratamiento (10). Como hipótesis clave en el primero de ellos se mantiene que, ante los sentimientos hostiles que dirigen los progenitores al niño en determinados períodos críticos evolutivos (durante los 6 primeros meses de vida, al iniciar las interacciones con objetos; desde los 6 a los 9 meses, cuando comienza el lenguaje, y, de los 18 a los 24 meses en que adquiere cierto control sobre su entorno), éste se retira, al percibir el mundo como un lugar peligroso. Carente de base empírica, dicha hipótesis ha sido duramente criticada desde diversas perspectivas por diferentes investigadores $(11 ; 12)$, pues, sin negar la mayor responsabilidad de las figuras adultas en el temprano desarrollo evolutivo y emocional del niño, es también conocida la influencia decisiva que la prole ejerce en los padres, cuyos estilos afectivos de interacción, a nivel cualitativo como cuantitativo, son igualmente influenciados por sus vástagos. Y es que, bajo tal bidireccionalidad, lo que subyace es la singular e irrepetible interacción diádica que padre (o madre) o quien actúe como tal establece con cada uno de los miembros de la fratría, conformando así diversos holones familiares. 
A Bettelheim (9) se le ha objetado también el uso tan impreciso que realizó del término psicosis infantil, al referirse con él a un extenso abanico de enfermedades mentales como la esquizofrenia, el autismo, determinadas formas de anorexia mental y ciertas toxicomanías de las organizaciones psicóticas presentes en los sujetos aquejados de encefalopatías. Es decir, para este autor, todo niño encerrado en sí mismo y rechazado por su entorno social próximo es psicótico (13).

Sea como fuere, la focalización del estudio del autismo en la esfera afectiva resultó efímera, pues en los años 60 del siglo pasado surgieron otras líneas de trabajo que suscitaron nuevos interrogantes, aportando también más dosis de esperanza, al amplificar las vías para describir y explicar el mencionado trastorno. Entre tales líneas novedosas figuraba la emprendida por Rutter (14), para quien el autismo se debía principalmente a alteraciones en el desarrollo del lenguaje, hipótesis explicativa que con el paso del tiempo se mostró insuficiente, ocasionando un acalorado debate entre los estudiosos del autismo, dadas las posiciones enfrentadas que existían entre unos y otros a la hora de determinar los síntomas primarios que definen y caracterizan este trastorno. Posteriormente, merced a la aparición de nuevas técnicas de exploración neurológica, neuropsicológica, neurofisiológica, etc., junto a aportaciones provenientes del terreno de la terapia de conducta, se produjeron algunos avances significativos en el estudio del autismo, a pesar de lo cual aún ignoramos aspectos centrales vinculados con su etiología, así como el papel mediador, influencia e importancia que poseen las diferentes constelaciones de síntomas en el trastorno autista (8).

\section{Concepto y diagnóstico diferencial del autismo: hitos históricos más señeros}

La primigenia concepción del autismo formulada por Kanner (3) sufrió al igual que otros cuadros clínicos diversas reformulaciones, debido a las dudas razonables que surgían al formular un diagnóstico, al competir por ejemplo el del autismo con otros como el de demencia precoz, esquizofrenia infantil o demencia infantil (15). De ello se infería que, cualquiera que fuera la etiqueta diagnóstica seleccionada, el autismo se encuadraba dentro de la categoría de los trastornos mentales, y no como acontecería más tarde en el campo de la deficiencia, al juzgarse en el primer caso que lo patognomónico residía en la incapacidad para relacionarse adecuadamente con otros, primando las deficiencias socioafectivas sobre las de carácter lingüístico y cognitivo, sucediendo de forma inversa en el segundo caso en donde cobraban un mayor peso etiopatogénico los déficits cognitivos sobre los de naturaleza emocional. Con todo, en la década de los ochenta del siglo XX logró alcanzarse un consenso en cuanto a la concepción del autismo como un síndrome conductual que afecta a una amplia gama de áreas tanto del desarrollo 
cognitivo como del afectivo, juzgándolo como un trastorno generalizado del desarrollo, tal como recogen las diferentes clasificaciones nosológicas (DSM-III, CIE-10 y DSM-IV-R). En 1990, Baird y su equipo proponen sin embargo abandonar la etiqueta diagnóstica de trastorno generalizado del desarrollo, considerando más adecuada la de trastorno específico del desarrollo, lo que para algunos sólo responde a razones de tipo clínico y no científico (8), pareciéndole a otros más correcto el término trastornos penetrantes del desarrollo (16).

Dejando a un lado tales cuestiones, pasaremos a referir algunos de los rasgos más sobresalientes del autismo, comenzando nuestro recorrido por las alteraciones de la conducta social dentro de la cual -dada la gran variabilidad que exhiben unos niños y otros- algunos autores han establecido diferentes subtipos de autismo, usando para ello como criterio de clasificación si la alteración social predominante recae en factores conductuales, en habilidades cognitivas o en las propias características sociales de estos niños (8). Bajo tales deficiencias cognitivas, algunos postulan que existe un severo problema lingüístico global que afecta a la comprensión y al uso de todas las formas de lenguaje, mientras que otros creen que lo que subyace es un déficit central que interfiere en la codificación de los estímulos y la formación de conceptos, derivándose en uno u otro caso la imposibilidad de establecer relaciones sociales adecuadas (17). Así pues, el alcance, grado y profundidad de las dificultades sociales estarían muy influidos por las destrezas cognitivas y lingüísticas que el individuo con autismo posea, de tal suerte que la evitación del contacto social pudiera deberse a la falta de competencias y habilidades sociales y no a una conducta deliberada de evitar relacionarse con otros (18).

En todo caso, lo que resulta evidente es que la desigual y genuina constelación que cada una de tales deficiencias conforma en la singularidad del autista hace que las alteraciones sociales de estos sujetos posean ingredientes distintivos en unos y otros, compartiendo no obstante como rasgo común el uso instrumental que suelen realizar de otras personas como medio para alcanzar sus fines (19). Los cambios en el entorno, por otro lado, originan una tenaz resistencia en el autista, como así se evidencia en la gran hipersensibilidad que exhibe al cambio, en un intento de preservar la invarianza del ambiente, reasegurando tal constancia mediante el despliegue de conductas rituales, a las que puede dedicar gran cantidad de tiempo.

En cuanto a las fallas en el lenguaje, cabe señalar que es posiblemente el rasgo evolutivo que antes se advierte en el círculo familiar y próximo del niño autista, dadas las comparaciones que los padres o cuidadores suelen establecer entre el desarrollo lingüístico y social de aquel con el de otros hermanos o niños conocidos, desfase del que aún toman mayor consciencia alrededor del año y medio o dos años, cuando otros niños llevan a cabo progresos rápidos en la adquisición del lenguaje y las conductas simbólicas. Es oportuno señalar que, aun cuan- 
do el niño autista adquiera un cierto desarrollo del lenguaje, sus patrones comunicativos diferirán cualitativamente del de los niños normales o del de aquellos afectos de trastornos del habla, estimando algunos estudios que entre un $28 \%$ y un $61 \%$ de casos nunca adquirirá un lenguaje expresivo (20), situando la comunidad científica tal porcentaje alrededor del $50 \%$ de los niños autistas. Conviene, no obstante, distinguir dentro del espectro autista quienes no hablan por su limitación intelectual de los que sin producir habla espontánea -pero ayudados de los programas específicos adecuados- podrían aprender algún sistema de comunicación no verbal, al no ser la falta de lenguaje oral sustituida espontáneamente por gestos en tales sujetos (21). Como alteraciones lingüísticas más comunes que corroboran dificultades pragmáticas figuran la inversión pronominal, mediante la que el niño al demandar algo se refiere a sí mismo usando el tú o el él; la ecolalia, que puede ser inmediata o bien demorarse horas o días (ecolalia retardada), evidenciando así su ausencia de sentido y de desconexión con la temática comunicativa presente, dando la impresión más de hablar $a$ alguien que con alguien (1). Cabe indicar también que, mientras la ecolalia inmediata está relacionada con la comprensibilidad del estímulo verbal, las variables que afectan la ecolalia retardada aún se ignoran, habiéndose hallado no obstante que ante un estímulo frente al cual el sujeto no posee respuesta, éste contestará reproduciendo toda o una parte del estímulo $(22 ; 23)$. Trabajos posteriores $(24 ; 25)$ han evidenciado también que ambos tipos de ecolalia, en contra de ideas anteriormente formuladas, constituyen actos lingüísticos comunicativos, en los que en muchos casos se corrobora una manifiesta comprensión, habiendo sido identificadas entre otras funciones que ejercen las de petición, protesta, afirmación, declarativo, llamada y autorregulación, permitiendo así ser encuadrados en un continuo de conducta que iría desde lo automático a lo intencionalmente comunicativo. Tal abanico de funciones, siempre interpretadas por el oyente, no evita seguir juzgando la ecolalia inmediata o demorada como parte de un uso estereotipado y repetitivo del lenguaje, fruto de una competencia comunicativa limitada, acerca de la que todavía se precisa realizar un mayor número de estudios longitudinales (21). Debe recordarse también que la ecolalia, a diferencia de otras conductas, forma parte del desarrollo lingüístico normal de un sujeto hasta alrededor de los 30 meses de edad, juzgándose patológica si persiste más allá de los 3-4 años (8).

Otras carencias comunicativas vinculadas al lenguaje expresivo que aqueja al niño autista aluden a alteraciones fonológicas, semánticas, defectos en la articulación, monotonía y labilidad en el timbre y en el tono de voz, así como reiteración obsesiva en las preguntas. En cuanto al lenguaje receptivo, presenta dificultades para atender y percibir la información, bajo nivel de comprensión gestual, pudiendo así evidenciarse mayores o menores discrepancias entre el lenguaje verbal y no verbal, muecas, tics y estereotipias, además de alteración o falta de contacto ocu- 
lar. Junto a tales fallas en el lenguaje expresivo o receptivo, puede darse también la coexistencia de limitaciones y peculiaridades tales como un cierto grado de sofisticación, carencia de emoción, imaginación y literalidad. Respecto a esta última, al captar las palabras en su sentido literal, los afectos de autismo son incapaces de percibir el simbolismo representacional que subyace en aquellas, de ahí la ecolalia, metalalia e inversión pronominal que manifiestan (13).

Como corolario de todo lo anterior podría afirmarse que lo que subyace bajo el trastorno autista es un grave déficit cognitivo, que abarca el lenguaje y las funciones de orden superior vinculadas a él, cuyo origen último habría que buscarlo en disfunciones cerebrales orgánicas. Como causa de estas últimas se ha especulado que en el proceso de desarrollo normal cerebral, en donde se alternan períodos de crecimiento y períodos de reducción, merced a los cuales se originan reorganizaciones cerebrales, es posible que haya un defecto de reducción en el autismo, en cuyo resultado se vean en mayor o menor grado comprometidas otras regiones cerebrales, en otros períodos del desarrollo. Si esto fuera así, los déficits en función ejecutiva de los autistas, posibles responsables de la conducta repetitiva y las dificultades de control de la atención, más evidentes en edades tardías que en las tempranas, podrían explicarse por un retraso o alteración del proceso madurativo del córtex prefrontal, una de las regiones cerebrales que tardan más en desarrollarse, lo que tal vez produciría una pérdida gradual de sinapsis, más que la formación de otras nuevas, y el reforzamiento de las conexiones sinápticas ya existentes, todo lo cual aún ha de ser verificado (7). Sea como fuere, como limitación cognitiva fundamental del sujeto autista se hallaría la profunda incapacidad para reducir la información mediante la extracción adecuada de características cruciales, tales como las reglas y las redundancias (26), como así han constatado las investigaciones en que como tareas experimentales se han usado la referenciación cruzada (en donde el sujeto ha de responder de forma selectiva a una de dos dimensiones) y la clasificación doble (en la que se indaga la capacidad de relacionar los sustantivos -objetosmediante el uso de preposiciones), siendo necesario para la primera realizar un análisis de los atributos de los objetos en sí mismos, mientras que para la segunda hay que analizar la oración para responder correctamente. Asimismo, si se coteja el desarrollo lingüístico normal de un niño con el de un autista, puede observarse que inicialmente el primero usa los objetos y el lenguaje de forma funcional, para después hacerlo de forma representacional, lo que no sucede en el segundo, cuyas capacidades funcionales están ya afectadas (27).

En lo que respecta a las alteraciones motoras, figuran los patrones de conducta repetitivos y estereotipados, cuyo objetivo fundamental aparentemente es proporcionar una retroalimentación sensorial o cinestésica, de carácter predominantemente visual y auditivo. Junto a ello también pueden aparecer trastornos del sueño, de los esfínteres y rechazo alimenticio (19). La antes aludida conducta 
autoestimuladora ejerce un papel crucial en la vida cotidiana del autista, dada la gran inversión de tiempo que gasta en su ejecución, abarcando desde comportamientos vinculados con la motricidad fina (v. g. observar la mano en cierta postura) hasta otros más alejados de su propia corporalidad como es el de contemplar ensimismado un objeto giratorio. La viscosidad y rigidez con que el autista se resiste a abandonar tales conductas ha sido interpretada por algunos como las responsables de interferir en la adquisición de conductas normales, al limitar su responsividad a aquellos estímulos implicados en la conducta estereotipada y no a los que conforman parte del entorno inmediato $(3 ; 28 ; 29)$.

Para explicar la variabilidad de tal responsividad, se ha recurrido al fenómeno conocido como hiperselectividad estimular, con el que quiere expresarse la peculiar tendencia que los autistas manifiestan para seleccionar un aspecto trivial del estímulo, ignorando el resto. Se ha postulado también que la citada hiperselectividad podría estar implicada en los problemas vinculados con la adquisición y generalización de nuevas conductas, así como en el aprendizaje mediante métodos tradicionales de soporte y en el modelado y aprendizaje social (30).

De naturaleza y consecuencias más dramáticas son las conductas autolesivas, mediante las cuales el autista se infringe daño a sí mismo, ocasionando en los casos más graves la necesidad de contención física, que si resulta prolongada (haciendo así que los miembros superiores e inferiores no se utilicen), produzca desmineralización, acortamiento de los tendones, detención del desarrollo motor, etc. (31). A ello también se une el desgaste emocional que implica para sus figuras de apego y cuidadores, como la merma que supone en sus posibilidades de integración socioeducativa.

En cuanto a los procesos cognitivos, abandonada la concepción del autismo como un trastorno fundamentalmente de carácter socio-afectivo, las investigaciones se han aglutinado en torno al estudio de anomalías en el procesamiento sensoperceptivo, así como en las deficiencias intelectuales. Respecto a estas últimas, parece ser que los autistas obtienen en tests que miden habilidades manipulativas o viso-espaciales y memoria automática un rendimiento significativamente mayor que en las pruebas donde las tareas requeridas exigen un procesamiento secuencial. Tal resultado, a nuestro entender, casaría con la idea ya referida acerca de la total irresponsividad que el autista manifiesta a aquellos estímulos ambientales distintos a los que participan en su conducta estereotipada, impidiéndole así desplegar la flexibilidad necesaria para ejecutar adecuadamente tareas de procesamiento secuencial. Relacionado también con ello está el debate que mantienen expertos para determinar si los déficits atencionales son la causa o bien la consecuencia de un fallido procesamiento perceptivo de la información estimular, habiéndose encontrado evidencia empírica a favor de la primera hipótesis $(32 ; 28)$. En todo caso, sea cual fuere la relación causal existente entre deficiencias atencionales y perceptivas 
en el autismo, lo que presentan sus afectados es un déficit sensorial aparente, al no haber sido éste corroborado a nivel de receptor, tal como sucede en la sordera (30). A tal cuestión se añaden también los defensores y detractores de la hipótesis de la preferencia sensorial $(33 ; 34)$, con la que se pretendía explicar la escasa eficacia de los autistas en el uso de la vista y el oído como canales propios de la comunicación, al preferir por encima de aquellos al tacto, olfato y gusto, primando así los estímulos que se sirven de receptores proximales, aparentemente propios de los primeros estadios evolutivos, sobre aquellos que lo hacen de receptores distales, más usados en estadios evolutivos posteriores. Datos experimentales, sin embargo, corroboran que el uso excesivo del gusto, tacto y olfato no se asocia al autismo de forma específica, sino a la edad mental baja (7), siendo así posible también que los autistas presenten déficit en el procesamiento de estímulos que se sirven de vías proximales, dada su aparente insensibilidad al dolor o al frío (35).

A los autistas también se les confiere un déficit conocido como ceguera mental, con que se expresa la incapacidad para atribuir e inferir estados mentales, en especial los que implican representaciones (36), al que se acoge bajo la llamada teoría de la mente, según la cual carecerían de las guías conceptuales para interpretar y pronosticar las conductas ajenas, viéndose así comprometido el desarrollo de sus competencias comunicativas, desde que todo acto conversacional es siempre un intercambio de ideas mutuamente relevantes en diferentes contextos de interacción simbólica que exige permanentemente colocarse en la piel del otro (16).

Referidas las áreas afectadas en el desarrollo psico(socio)afectivo de los autistas, abordaremos lo relativo al diagnóstico, donde los principales sistemas de clasificación nosológica, DSM-IV-R y CIE-10 han reagrupado los criterios diagnósticos previos en tres de naturaleza comportamental y otro de carácter cronológico. Como resultado de ello, y a diferencia de lo que se establecía en el DSM-III-R, actualmente se exige para formular el diagnóstico de autismo que una de las tres áreas alteradas (conducta social, comunicación o juego simbólico) muestre un retraso o desviación de lo acordado como desarrollo normal antes de los 36 meses de edad (autismo de inicio en la infancia) y no después de tal período como se admitía también antes (autismo de inicio en la niñez). Asimismo, a la hora de evaluar el grado y alcance de las conductas psicopatológicas, junto a criterios cuantitativos se han añadido otros de carácter cualitativo, como son que en el área de las relaciones sociales se juzgue más el cómo que el cuánto del déficit social, o que en las fallas comunicativas se valore tanto el retraso en el desarrollo del habla como la desviación o déficit cualitativo de las conductas implicadas en la comunicación (8), considerando todo el conjunto como un proceso exploratorio continuado, guiado por criterios funcionales, y donde la historia evolutiva del sujeto sirve de marco de referencia para la intervención presente y futura (21). Al respecto cabe afirmar que, incluso en los casos en que el niño autista adquiera lenguaje hablado, según algu- 
nos trabajos, el desarrollo evolutivo más favorable se producirá en los menores que nunca presentaron una falta profunda de respuesta a los sonidos, adquiriendo el habla útil en torno a los cinco años y en donde la fase de ecolalia resultó transitoria (37). Asimismo, dada la variabilidad del grupo de autistas hablantes, que oscila desde los que usan palabras o frases para regular su conducta, gestos para informar de algo, ecolalias para iniciar o mantener interacciones sociales y ninguna vía para expresar estados de ánimo, resulta crucial diseñar contextos naturales para alentar las interacciones comunicativas, siendo la enseñanza incidental una herramienta clave para ello, pues, además de ser eficaz para favorecer/incrementar generalizaciones de lo aprendido, confiere más iniciativa al propio niño. De igual modo, los problemas de conducta que surjan deberán integrarse en el programa diseñado para el aprendizaje de habilidades comunicativas (21).

Seguir tales indicaciones no evita sin embargo la dificultad de establecer un diagnóstico diferencial del que no emanen dudas razonables entre el autismo y otros trastornos que, aun compartiendo algunos síntomas comunes, no cumplen el conjunto de criterios diagnósticos consensuados por la comunidad científica (38). Es posible también que el trastorno autista sea en realidad el resultado de una patología específica preexistente como la rubéola congénita, esclerosis tuberosa, encefalopatía, lipoidosis cerebral o neurofibromatosis o que se halle asociado a otros trastornos como el síndrome de Down o a crisis epilépticas que acontecen durante la adolescencia.

Aun así, la dificultad mayor que afrontan quienes han de emitir un diagnóstico de autismo radica en los síntomas primarios o rectores que comparten tal trastorno con otros como la esquizofrenia infantil, disfasia evolutiva, retraso mental, privación ambiental, síndrome de Rett, síndrome de Asperger y trastornos infantiles desintegrativos. Sirva para comprender ello que, la esquizofrenia infantil, a diferencia del autismo, es de inicio más tardío (después de los cinco años de edad), presentando sus aquejados una historia familiar de psicosis, además de alteraciones del pensamiento (delirios), de la percepción (alucinaciones), déficits psicomotrices y pobre salud física (38). Tal distinción se refleja también en el plano psicoterapéutico, en donde mientras la esquizofrenia responde mejor al tratamiento psicofarmacológico, el autismo lo hace con técnicas de modificación de conducta $(39 ; 40)$. De forma análoga, existen semejanzas y diferencias entre el autismo y el resto de los trastornos antes mencionados.

\section{Desentrañando el enigma: hipótesis explicativas formuladas en torno al autismo}

Tradicionalmente suelen diferenciarse dos grupos según los factores a investigar, distinguiéndose dentro del primero los que estudian factores genéticos, cro- 
mosómicos y variables neurobiológicas; mientras que dentro del segundo se hallan las que rastrean los ingredientes afectivos, cognitivos y sociales subyacentes al comportamiento autista. Lo importante, en todo caso, será conocer con la mayor exactitud posible si los déficits ocasionados en el sujeto le impedirán alcanzar el desarrollo normal del sistema neurológico en un periodo crítico (7).

En cuanto a los factores genéticos, existen hallazgos que apuntan a la presencia de diversas anomalías en el cariotipo de algunos autistas, en quienes se han detectado alteraciones en gran parte de los pares cromosómicos, con excepción de los pares 7, 14, 19 y 20. Asimismo, y aunque ya fue referida por H. A. Lubs en 1969 , sin que sus resultados hasta la fecha sean definitivos, se manifiesta en algunos cuadros autistas el llamado síndrome X-frágil, según el cual hay una ausencia de sustancia en el extremo distal del brazo largo del cromosoma X que afecta a ambas cromátides. Otras investigaciones constatan la mayor incidencia del trastorno autista al comparar la frecuencia de éste entre hermanos y gemelos monocigóticos respecto a la población general (8).

Parece ser también el virus de la rubéola el proceso infeccioso que más se ha detectado en casos de autismo, habiéndose también descrito otros asociados a infecciones intrauterinas y posnatales originadas por distintos virus (citomegalovirus, sífilis, herpes simple, etc.). De ello algunos expertos han inferido la posibilidad de una alteración en el sistema inmunológico de los niños autistas, causada previsiblemente por los linfocitos $\mathrm{T}$, que, al presentar defectos genéticos, reducirían la resistencia del feto a los ataques víricos. Junto a la mayor susceptibilidad del feto a la viriasis se ha formulado también -como origen del déficit inmunitario- la exposición del feto al virus en una etapa muy temprana de la diferenciación inmunológica, conjeturando algunos que el autismo infantil pudiera ser en realidad un trastorno autoinmune.

Dentro de las alteraciones metabólicas, por el contrario, la que ha encontrado mayor respaldo empírico es la producida por la fenilcetonuria, cuya asociación con el autismo refirió ya Friedman en 1969, en cuya muestra había un 92\% de casos con tal disfunción metabólica.

Asimismo se ha evidenciado hiperserotoninemia en cuadros de autismo como en diversos trastornos sin tal sintomatología asociada, de tal suerte que, aun consiguiendo una disminución del nivel de 5-HT plasmático no se ha corroborado una mejoría de la conducta autista. Con todo, lo que resulta incuestionable es el papel determinante que la alteración de la 5-HT ejerce en la producción de los trastornos del desarrollo, al participar entre otras tareas en la neurogénesis de los primeros meses de vida embrionaria (41). Otra hipótesis postula que el autismo sería el resultado de un fallo en el metabolismo de una de las aminas biógenas, lo que, dada la inconsistencia de los hallazgos obtenidos, resulta insostenible. De igual forma se ha hipotetizado sobre el posible efecto alucinógeno de las aminas bióge- 
nas, desde que se han hallado concentraciones de bufotenina en la orina de niños autistas, aunque se ignora si la citada sustancia se gesta en el cerebro o en algún otro tejido corporal. Asimismo han sido cuestionados los procedimientos de saturación, al no haberse observado cambios significativos en los patrones conductuales de los autistas tras administrarles durante un tiempo una amina biógena precursora capaz de precipitar los efectos alucinógenos (42).

Otras críticas a los estudios neurobiológicos aluden a la necesidad de investigar con grupos homogéneos, al participar de forma desigual en ellos tanto autistas como otros sujetos etiquetados como psicóticos, esquizofrénicos, hospitalizados y retrasados medios y severos. Más aún, dentro de la categoría de autismo conviven en ocasiones diversos subgrupos que pueden tener diferentes etiologías $(43 ; 44)$, por lo que se ha sugerido la necesidad de establecer una posible jerarquía de aquellas, comenzando para ello por los casos relativamente raros de trastorno autista realmente heredado, y siguiendo con los más comunes de autismo genéticamente inducido (vinculados con un daño intrauterino muy temprano); finalizando tal listado con un número significativo de casos en donde un daño perinatal o tal vez posnatal ha replicado el defecto genético aludido en los dos grupos anteriores (45).

En cuanto a hipótesis neurológicas, puede referirse la teoría de la disfunción del sistema dopaminérgico formulada por A. R. Damasio y R. G. Maurer en 1978 (7), como la disfunción cortical primaria como factor causante del autismo y la disfunción cerebral primaria del tronco cerebral como variable etiopatogénica. Asimismo, no hay pruebas concluyentes de que los niños autistas estén crónicamente en un estado de infra o hiperactivación, ni que el SAR (sistema activador reticular) esté implicado en el autismo, pues es posible que un estado de activación anormal sea secundario a otro factor tal como la estimulación ambiental (46; 15; 47). Casi diez años después, Sahley y Panksepp postularon que el aislamiento autista estaría relacionado con un exceso de péptidos, sustancias similares al opio producidas de forma endógena por el cerebro y generadoras de efectos placenteros, cuya liberación por ejemplo acontece en un niño cuando su madre le brinda atención y mimo, lo que no sucedería en el menor autista, que sin necesidad de involucrarse en tal relación con su figura materna ya libera en exceso tal opiáceo endógeno. Estudios rigurosos igualmente corroboran que tras la administración de una sustancia que bloquea los efectos de esos opiáceos naturales se mejoran en algunos casos los síntomas autistas, disminuyendo notablemente las autoagresiones (16).

Más recientemente se ha formulado también que una disfunción en el sistema de neuronas espejo (SNE), halladas en el cortex premotor ventral -pares opercularis en el giro frontal inferior (área 44 de Brodmann) - y en el sector rostral del lóbulo parietal inferior, cuya activación tiene lugar al observar las conductas de otros o la ejecución de las propias, podría ser la causante de los déficits sociales presentes en el autismo, y por extensión de la inadecuación o falta de respuesta 
emocional a las distintas exigencias sociales, dada la posible mediación del antes referido SNE, junto al sistema límbico, en la observación, imitación y comprensión de las expresiones y estados emocionales de otros sujetos realizadas por autistas (48). Y es que, de inhibirse tal SNE resultaría imposible para un individuo captar el significado subjetivo que confiere a la emoción percibida en otro, mostrándose también incapaz de imitar los ingredientes conductuales vinculados a tal estado emocional (49). Con todo, a pesar de las diferentes hipótesis planteadas, es razonable pensar, en línea con planteamientos ya antes expresados, que lo que realmente subyaga bajo el trastorno autista sea una constelación de diversas causas etiopatogénicas, de ahí la complejidad de su abordaje y el amplio abanico de distintos subtipos de autismo que pueden encontrarse.

En lo que a hipótesis psicológicas explicativas se refiere, desde las primitivas formulaciones culpabilizadoras de Kanner (3), para quien el trastorno autista era el resultado de una interacción fallida y emocionalmente fría de los progenitores con sus vástagos, que posteriormente modificó, concibiéndolo como un fenómeno biosocial en donde predisposiciones constitucionales interactúan adversamente con condiciones sociales (50) o bien eran la expresión corporeizada en el sujeto autista de una marcada predisposición familiar al aislamiento social (51), se ha girado, al igual que en la psicología académica, hacia planteamientos centrados en los aspectos cognitivos que intervienen y gobiernan nuestro psiquismo, en detrimento de los ingredientes afectivos, que parecen haber sido relegados a un segundo plano, primando las aportaciones de los modelos cognitivos sobre el resto de las demás. Esto no ha impedido, sin embargo, que la pretérita teoría socioafectiva postulada por Kanner (3) haya sido replanteada por Hobson $(52 ; 53 ; 54 ; 55)$, al igual que la teoría cognitiva clásica, que ha pasado a denominarse teoría cognitivo-afectiva del autismo. Conjugando ambas perspectivas, Rivière (56) se ha servido de la noción de intersubjetividad secundaria, dentro de la que incluye tanto un componente afectivo (la motivación de compartir afectivamente experiencias) como un componente cognitivo (al concebir al autista como sujeto de experiencia).

De forma sintética, lo que sostiene la teoría de Hobson (57) es la falta de componentes constitucionales en los autistas para interactuar emocionalmente con otras personas, lo que les impide configurar un mundo propio y común, así como ser incapaces de reconocer que los otros poseen sus propios pensamientos, sentimientos, etc. Es decir, carecen de la capacidad de abstraer, sentir y pensar simbólicamente. En línea con esto último, la teoría cognitiva clásica postula que los problemas sociales y de comunicación presentes en el autismo podrían estar causados por una alteración de la capacidad metarrepresentacional o uso de representaciones de segundo orden, que al faltar en los sujetos autistas haría que éstos manifestaran dificultades en comunicación preverbal (atención conjunta y actos protodeclarativos), juego simbólico, habilidades pragmáticas del lenguaje, empatía y 
otros aspectos del funcionamiento social $(58 ; 59 ; 60)$. Es cierto, no obstante, que existen igualmente habilidades/destrezas que no implican metarrepresentaciones, como son las comunicativas prelingüísticas o las habilidades para evaluar el significado de las expresiones afectivas, que también están alteradas, siendo por tanto posible que haya mecanismos diferentes al antes señalado metarrepresentacional alterados previamente (21).

Por su parte, la hipótesis cognitivo-afectiva argumenta el carácter primario del déficit afectivo como de su homólogo cognitivo, a los que atribuye las dificultades encontradas al percibir y tomar consciencia de los estados mentales y emocionales de otras personas. Entre las críticas que ha recibido la teoría cognitivo-afectiva figura la de que los autistas son incapaces de percibir contingencias, lo que implicaría que tales sujetos son difíciles de condicionar, algo que numerosas investigaciones refutan. En su defensa, quienes apuestan por la citada teoría descartaron más tarde la hipótesis del procesamiento de contingencias, haciendo responsable de los fallos detectados en la atención gestual conjunta a un déficit en la regulación de la activación, el cual alteraría la comprensión del valor del afecto como señal, repercutiendo en la comprensión de los estados mentales y afectivos de otros (61).

Ante tal diversidad de hipótesis psicológicas, en las últimas décadas la investigación se ha desviado hacia la búsqueda de una sola alteración esencial capaz de explicar adecuadamente el amplio conjunto de anomalías y desfases evolutivos que, a nivel intrasujeto como intersujeto, manifiestan los autistas, línea de trabajo cuya idoneidad o no en último término serán los propios datos empíricos la que la determine. Por el contrario, al ser en la actualidad todo tratamiento del autismo de carácter sintomático, dada la imposibilidad de intervenir directamente sobre el curso etiopatogénico que genera el síndrome, y a que se ignoran las intervenciones eficaces, aun admitiendo ciertos principios generales muy abstractos, todo tratamiento ha de ser extremadamente individualizado e integrado (56).

\section{Evaluación e intervención en el autismo: niños, familia y cuidadores importan}

Evaluar el autismo hasta mediados de los años ochenta del pasado siglo requería una evaluación conductual previa a la exploración cognitiva del sujeto, dada la gran dificultad que el uso de pruebas estandarizadas de inteligencia conllevaba en tal población clínica (62), lo que forzó a investigadores y clínicos a agudizar el ingenio y plantear procedimientos de evaluación alternativos a los hasta entonces existentes. Fruto de tal esfuerzo intelectual surgieron dos tipos de escalas conductuales específicas para explorar el autismo, unas con fines diagnósticos como la Diagnostic Checklist for Behavior Disturbed Children y el British Working Party 
Diagnostic System, y otras con objetivos principalmente descriptivos, como eran la Behavior Evaluation Scale (BES), la Adaptative Behavior Scale y la Vineland Social Maturity Scale. Ha de indicarse, sin embargo, que tales escalas diagnósticas resultan de más utilidad si están acompañadas de otras pruebas que evalúen el comportamiento adaptativo o el nivel/perfil psicoeducacional. En lo que concierne a las escalas descriptivas, pueden proporcionarnos información muy relevante de los déficits conductuales y nivel de funcionamiento del sujeto (30).

En cuanto a nuestro país, fruto del trabajo de la Asociación de padres de niños autistas de Guipúzcoa (GAUTENA), puede consultarse la adaptación de la primera versión de la Childhood Autism Rating Scale (1980) (CARS), de la que en 1988 surgió otra versión mejorada, paliando así la escasa precisión en la formulación de sus ítems (63), estando también disponible la Autism Behaviour Checklist (ABC), que forma parte del Autism Screening Instrument for Educational Planning (ASIEP), de enorme utilidad para profesionales y profesores de educación especial, al igual que el Test de Evaluación del Desarrollo Preescolar y Especial (TEDEPE). Muy extendidos también en la praxis clínica y educativa es la Autism Diagnostic Interview Revised (ADI-R) (64), como los cuestionarios retrospectivos aplicados a padres tal como la Lista de Diagnóstico del Autismo (1988) (LDA), mientras que para el diagnóstico precoz se han elaborado cuestionarios como el Checklist for Autism in Toddlers (CHAT) (21).

Vinculado a ello está el desacuerdo manifiesto que presentan investigadores y clínicos respecto a la prevalencia del autismo, al juzgar que la supervisión exclusiva de los sujetos institucionalizados no es el sistema más idóneo para saber qué porcentaje de población se siente aquejada por este trastorno generalizado del desarrollo, al conocerse casos de autistas que están fuera de tales registros oficiales.

Respecto a la intervención, conviene referir las prometedoras expectativas que en la última década del siglo XX suscitó la comunicación facilitada, mediante la cual aparentemente se incrementaba la producción lingüística autística, aunque no de forma espontánea, sino (como evidencian distintos estudios serios por la acción del facilitador), que, a través de una orientación sutil de la muñeca, el brazo o el hombro cuando el dedo del niño autista estaba sobre el teclado, era quien realmente indirecta pero absolutamente inconsciente generaba el mensaje (7).

Precisamente, además del programa de comunicación total, para la enseñanza de destrezas comunicativas y el despliegue de lenguaje espontáneo de niños autistas en diferentes contextos naturales se halla el método TEACCH (Teaching Spontaneous Communication to Autistic and Developmentally Handicapped Children), mediante el cual, sirviéndose del lenguaje verbal como de modalidades no orales, se ofrece una guía de objetivos y actividades, más que propiamente una programación, en la que aparecen sugerencias acerca de cómo evaluarlas y programarlas, distinguiendo cinco dimensiones en los actos comunicativos: la fun- 
ción, el contexto, las categorías semánticas, la estructura y la modalidad, que además de servir para programar los objetivos de desarrollo comunicativo en cada una de ellas, son claves importantes en el proceso de enseñanza/aprendizaje (16).

Para reducir las estereotipias, apoyándose en estudios empíricos realizados con mamíferos no humanos, donde se evidenciaba el decremento de aquellas inducidas por apomorfina o anfetaminas usando neurolépticos (como el haloperidol, tioridazina -meleril-, clorpromacina -largactil- y Risperdal, cuyos efectos secundarios son menores) se corroboró la eficacia psicoterapéutica de tales medicamentos en algunos sujetos y etapas, siempre y cuando las dosis fueran revisadas y la medicación se retirara tan pronto como fuera posible, evitando así efectos secundarios indeseables. Asimismo se ha utilizado fenfluoramina en autistas con elevados niveles de serotonina en sangre y pautas marcadas de hiperactividad y conductas estereotipadas, cuestionándose su eficacia real a medio y largo plazo. Desde la terapia conductual, los éxitos alcanzados han sido también discretos, incluyendo el arsenal terapéutico usando técnicas como el reforzamiento diferencial de conductas alternativas, la extinción sensorial de las consecuencias autoestimulatorias, la sobrecorrección y el castigo contingente de las conductas estereotipadas, cuyo uso ha sido criticado por su escasa eficacia, efectos indeseables e implicaciones éticas derivadas durante y después de su aplicación.

De tales resultados algunos han postulado que, al igual que sucede con los individuos normales, que manifiestan una relación directa entre las estereotipias y la falta de alternativas funcionales o de estímulos significativos en el medio, se les oferte a los autistas diferentes alternativas funcionales de actividad, merced a las cuales sus vidas adquieran el mayor sentido, posibilitándoles también acciones anticipatorias que incrementen su atención y motivación para realizar conductas funcionales, siendo entonces capaces de desarrollar competencias e instrumentos de comunicación. Junto al descenso en la probabilidad, frecuencia, intensidad y duración de las estereotipias, ello ha influido en las estrategias actuales de tratamiento, que han pasado desde la extinción de tales conductas juzgadas negativas y de carácter autoestimulatorio, presentes por así decir en un individuo depravado, a la construcción de conductas positivas y funcionales capaces de producir desarrollo (56).

Otro escollo diario al que se enfrentan quienes conviven y cuidan al autista es la rutina comportamental y ambiental que necesita, caracterizada por la rigidez, fijación obsesiva y fuerte resistencia al cambio, frente a la que, dada su imposible eliminación, se ha optado por reducir hasta donde sea posible, usándose como criterio el grado con que interfiere en las posibilidades de relación y aprendizaje de aquél.

En cuanto a las medidas implementadas para tratar la conducta autolesiva, en modo alguno privativa del autismo, están el uso de medicamentos, la contención física y el afecto tranquilizador, cuya eficacia psicoterapéutica se ha demostrado inferior a la alcanzada usando técnicas operantes como la extinción y el castigo 
(30). Y es que, extendiendo el uso de tales técnicas al entorno familiar, a través del adecuado entrenamiento a padres y otras figuras de apego, que así aliviaban su posible culpa y adoptaban una posición activa ante la condición autista de su hijo, se producía un cambio significativo en el abordaje y tratamiento, que de esta forma dejaba de estar circunscrito al ámbito clínico, para hacer copartícipes de él a otros, especialmente a los padres, cuya pretérita imagen negativa de causantes del autismo se desdibujaba, consolidándose progresivamente otra que los percibía como agentes facilitadores de cambios positivos en el niño y en la dinámica familiar, aunque sujetos como cualquier otro hogar a los vaivenes emocionales propios de todo grupo humano. Asimismo, más que instruir a los progenitores en determinadas técnicas, la experiencia clínica muestra que es más útil ayudarles a solventar problemas de la vida cotidiana, siendo para ello un elemento clave conocer con la mayor precisión posible el nivel actual y potencial de destrezas cognitivas del menor autista, para que así los objetivos y metas terapéuticos establecidos resulten adecuados y realistas (65).

Alcanzar tales logros precisa que padres, profesores y terapeutas implicados coordinen sus distintas parcelas de influencia y trabajo, dadas las grandes dificultades que los niños autistas manifiestan para comprender realmente lo que aprenden y para generalizar lo aprendido a otros contextos diferentes de aquel donde originariamente se produjo tal aprendizaje. Por ello se exige que el proceso de enseñanza-aprendizaje del autista tenga lugar dentro de un marco sistemático y estructurado de normas y pautas de conducta, cuyo diseño final vendrá determinado en función de la singularidad y necesidades requeridas por su perceptor.

Lo mismo puede decirse de su futura integración laboral, en donde exhibirá un mejor desempeño laboral en trabajos que requieran buenas habilidades visomotoras, destreza motora fina o razonamiento no verbal, por lo que resultará muy conveniente que antes de ello reciban una enseñanza vocacional, basada en tareas reales y no hipotéticas (por ejemplo ensamblar piezas de hierro y no de un puzzle) llevadas a cabo en su puesto de trabajo, para así inferir las posibilidades futuras del sujeto, como el grado de estructuración exigido para la actividad y las modificaciones o adaptaciones necesarias para realizar las tareas encomendadas. De igual forma, el personal especializado y responsable del autista en el entorno laboral debe observar los problemas de conducta y falta de habilidades sociales que pueda manifestar, al recaer en ambos y no en la ausencia de competencia laboral la potencial pérdida de empleo, siendo también útil generalizar a los autistas lo aprendido en los programas de habilidades sociales creados para retrasados mentales adultos en entornos de trabajo, debiendo asimismo valorarse las características personales que el contratante juzgue más relevantes para el puesto laboral a cubrir.

Todo ello, como es fácil comprender, está orientado a que el autista consolide su ocupación laboral, pareciendo entonces solaparse las diferencias entre los 
servicios dedicados a la adaptación laboral de los encauzados hacia el apoyo en la comunidad, dentro de la cual el autista, según su nivel de afectación y grado de autonomía, podrá vivir junto a su familia biológica, adoptiva o en residencias pequeñas (apartamentos o casas unifamiliares) lo más integrado y normalizado posible, alentándole para ello al máximo la participación en actividades y situaciones cotidianas. Complementando esto, debe llevarse a cabo una planificada supervisión a corto, medio y largo plazo, en la que puede ser imprescindible contar con profesionales cualificados que evalúen los logros y objetivos conseguidos (21).

\section{Reflexiones finales}

De todo lo expresado cabe inferir que, dada la falta de marcadores biológicos claros en el autismo, sólo puede definirse éste como un trastorno muy heterogéneo, lo que dificulta además del diagnóstico, la genuina y profunda comprensión de la condición autista y de los procesos psicológicos alterados de base (66), por lo que aún es absolutamente acertado lo que en 1993 ya expresó Rivière al decir que «el autismo es provisionalmente incurado, pero no definitivamente incurable» (67), dada la mejora en la calidad de vida que han experimentado las personas con autismo. Asimismo en la búsqueda de otras respuestas se profundiza en líneas de investigación como la de las neuronas espejo, añadiéndose así nuevas piezas al rompecabezas que es el trastorno autista. Esto, sin embargo, no debe hacernos bajar la guardia, como tampoco concebir falsas esperanzas, sino sólo continuar investigando con similar entusiasmo, de forma multidisciplinar, allanando así el angosto y tortuoso camino que aún debe recorrerse en la comprensión, explicación profunda y tratamiento del enigma llamado autismo.

\section{BIBLIOGRAFÍA}

(1) RutTer, M., «Diagnóstico y definición», en RutTer, M.; SCHOPLer, E. (coords.), Autismo. Reevaluación de los conceptos y el tratamiento, Madrid, Alhambra, 1984, pp. 1-26.

(2) Rivière, Á., «Trastorno de la flexibilidad en el autismo», en VALDEZ, D. (ed.), Autismo: enfoques actuales para padres y profesionales de la salud y la educación, 2, Buenos Aires, Fundec, 2001a.

(3) Kanner, L., «Autistic Disturbances of Affective Contact», Nervous Child, 1943, 2, pp. 217-230.

(4) Rivière, Á.; VALDEZ, D., «Autismo: definición, evaluación y diagnóstico», Módulo 1 2000, Buenos Aires, FUNDEC (Fundación para el desarrollo de los estudios cognitivos).

(5) Kanner, L., Child Psychiatry, Springfield, Illinois, C. C. Thomas, 1979. 
(6) Sanua, V. D.; Kanner, L., «The Man and the Scientist», Child Psychiatry and Human Development, 1990, 21 (1), pp. 3-23.

(7) Frith, U., Autismo. Hacia una explicación del enigma, Madrid, Alianza, 2004.

(8) Pérez, M.; Ramos, F., «El autismo infantil», en Belloch, A.; Sandín, B.; Ramos, F. (coords.), Manual de Psicopatología, 2, Madrid, McGraw-Hill/Interamericana de España, 1995, pp. 651-674.

(9) Bettelheim, B., La fortaleza vacía. Autismo infantil y nacimiento del yo, Barcelona, Paidós, 2001.

(10) Ruttenberg, B., «A Psychoanalytic Understanding of Infantile Autism and its Treatment», en Churchill, D. W.; Alpern, G. D.; Demyer, M. K. (eds.), Infantile Autism, Springfield, Ill., C. C Thomas, 1971.

(11) RutTer, M., «Concepts of autism», Journal of Child Psychology and Psychiatry, 1968, 9, pp. $1-25$.

(12) Wing, J. K., «Review of Bettelheim: 'The Empty Fortress'», British Journal of Psychiatry, 1968, 114, pp. 788-791.

(13) De Villard, R., Psicosis y autismo del niño, Barcelona-México, Masson, 1986.

(14) RutTer, M., «Diagnosis and Definition of Childhood Autism», Journal of Autism and Childhood Schizophrenia, 1978b, 8, pp.139-161.

(15) RutTer, M., «Etiología y tratamiento: Causa y curación», en RUTTER, M.; SCHOPLER, E. (coords.), Autismo. Reevaluación de los conceptos y el tratamiento, Madrid, Alhambra, 1984, pp. 310-318.

(16) RIVIÈRE, Á., Autismo. Orientaciones para la intervención educativa, Madrid, Trotta (2001b)

(17) WING, L., «Características sociales, comportamentales y cognitivas: enfoque epidemiológico», en RUTTER, M.; SCHOPLER, E. (coords.), Autismo. Reevaluación de los conceptos y el tratamiento, Madrid, Alhambra, 1984, pp. 27-44.

(18) Howlin, P., «Evaluación de la conducta social», en RUTTER, M.; SchOPLER, E. (coords.), Autismo: Reevaluación de los conceptos y el tratamiento, Madrid, Alhambra, 1984, pp. 60-66.

(19) De Ajuriaguerra, J.; Marcelli, D., Manual de Psicopatología del niño, Barcelona, Masson, 1987.

(20) Paul, R., «Comunication», en Cohen, D.; Donnelan, A. (comps.), Handbook of Autism and Pervasive Developmental Disorders, Nueva York, Wiley, 1987.

(21) Canal, R., Deficiencias sociales severas. Autismo y otros trastornos profundos del desarrollo», en Verdugo, M. A. (dir.), Personas con discapacidad. Perspectivas psicopedagógicas y rehabilitadoras, Madrid, Siglo XXI, 1995, pp. 1027-1115.

(22) Carr, E. G.; Schreibman, L; LovaAs, O. I., «Control of Echolalic Speech in Psychotic Children», Journal of Abnormal Psychology, 1975, 3, pp. 331-351.

(23) Schreibman, L.; Carr, E. G., «Elimination of Echolalic Responding to Questions Through the Training of a Generalized Verbal Response», Journal of Applied Behavior Analysis, 1978, 11, pp. 453-463.

(24) Prizant, B. M.; Duchan, J., The Function of Immediate Echolalia in Autistic Children, Journal of Speech and Hearing Research, 1981, 146, pp. 241-249.

(25) Prizant, B. M.; Rydell, P. J., «Analysis of Function of Delayed Echolalia in Autistic Children», Journal of Speech and Hearing Research, 1984, 27, pp. 183-192. 
(26) Hermelin, B., «Codificación y modalidades sensoriales», en WING, L.; Everald, M. P., y otros, Autismo infantil: Aspectos médicos y educativos, Madrid, Santillana, 1982, pp. 155-188.

(27) Menyuk, P., «El Lenguaje: problemas y causas», en RutTer, M.; SCHOPLER, E. (coords.), Autismo: Reevaluación de los conceptos y el tratamiento, Madrid, Alhambra, 1984, pp. 101-112.

(28) LovaAs, O. I.; Litrownik, A.; ManN, R., «Response Latencies to Auditory Stimuli in Autistic Children Engaged in Self-Stimulatory Behavior», Behavior Research and Therapy, 1971, 9, pp. 39-49.

(29) Rincover, A., «Sensory Extinction: A Procedure for Eliminating Self-Stimulatory Behavior in Autistic Children», Journal of Abnormal Child Psychology, 1978, 6, pp. 299-310.

(30) Schreibman, L.; Mills, J. I., «Autismo infantil», en Ollendick, Th. H.; Hersen, M. (coords.), Psicopatología infantile, Barcelona, Martínez Roca, 1986, pp. 176-212.

(31) LovaAs, O. I.; Simmons, J. Q., «Manipulation of Self-Destruction in Three Retarded Children», Journal of Applied Behavior Analysis, 1969, 2, pp. 143-157.

(32) Koegel, R. L.; Wilhem, H., «Selective Responding to the Components of Multiple Visual Cues by Autistic Children», Journal of Experimental Psychology, 1973, 15, pp. 442-453.

(33) GoldFarb, W., Childhood Schizophrenia, Cambridge, Harvard University Press, 1961.

(34) SCHOPleR, E., «Early Infantile Autism and Receptor Processes», Archives of General Psychiatry, 1965, 13, pp. 327-335.

(35) Frith, U.; Baron-Cohen, S., «Perception in Autistic Children», en Cohen, D. J.; Donnellan, A. M. (comps.), Handbook of Autism and Pervasive Developmental Disorders, 1987, Nueva York, Wiley.

(36) Baron-Cohen, S., «From Attention-Goal Psychology to Belief-Desire Psychology: the Development of Theory of Mind, and Dysfunction», en BARON-COHEN, S.; TAGER-FlusberG, H.; Cohen, D. (eds.), Understanding other Minds. Perspectives from Autism, Nueva York, Oxford University Press, 1993, pp. 59-82.

(37) Rutter, M., «Cuestiones evolutivas y pronóstico», en Rutter, M.; Schopler, E. (coords.), Autismo. Reevaluación de los conceptos y el tratamiento, Madrid, Alhambra, 1984, pp. 479-487.

(38) WING, L., «Diagnóstico, descripción clínica y pronóstico», en WING, L.; EveraLD, M. P., y otros, Autismo infantil. Aspectos médicos y educativos, Madrid, Santillana, 1982, pp. 32-82.

(39) Goldfarb, W., A Time to Heal. Corrective Socialization: A Treatment Approach to Childhood Schizophrenia, Nueva York, International University Press, 1969.

(40) Polaino, A., Introducción al estudio científico del autismo, Madrid, Alhambra, 1982.

(41) DíEz Cuervo, A., Modelos neurobiológicos del trastorno autista, Actas del VII congreso nacional de autismo, Salamanca, Amaru, 1993, pp. 85-104.

(42) Shaywitz, B. A.; Cohen, D. J., «The Neurochemical Basis of Infantile Autism», en Lockman, L. A.; Swaiman, K. F.; Drage, J. S., y otros (eds.), Workshop on the Neurobiological Basis of Autism, Bethesda, Md: National Institutes of Health Publication, 1979.

(43) Coleman, M., «Serotonin and Central Nervous System Syndromes of Childhood, a Review», Journal of Autism and Childhood Schizophrenia, 1973, 3, pp. 27-35.

(44) Coleman, M., «Introduction», en Coleman, M. (ed.), The Autistic Syndromes, Nueva York, Elsevier, 1976. 
(45) ORnITZ, E. M., «¿Homogeneidad o heterogeneidad biológica?», en RutTER, M.; SCHOPLER, E. (coords.), Autismo: Reevaluación de los conceptos y el tratamiento, Madrid, Alhambra, 1984, pp. 232-238.

(46) ORnitz, E. M., «Estudios neurofisiológicos», en RutTER, M.; Schopler, E. (coords.), Autismo: Reevaluación de los conceptos y el tratamiento, Madrid, Alhambra, 1984, pp. 113-135.

(47) Lovaas, O. I.; Koegel, R. L.; Schreibman, L., «Stimulus Overselectivity in Autism», Psychological Bulletin, 1979, 86, pp. 1236-1254.

(48) Dapretto, M.; Davies, M. S.; Pheifer, J. H., y otros, «Understanding Emotions in Others: Mirror Neuron Dysfunction in Children with Autism Spectrum Disorders», Nature Neuroscience, 2006, 9, 1, pp. 28-30.

(49) Iacoboni, M., «Neural Mechanisms of Imitation», Current Opinion in Neurobiology, 2005,15 , pp. 632-637.

(50) EisenberG, L.; KanNer, L., «Early Infantile Autism 1943-1955», American Journal of Orthopsychiatry, 1956, 26, pp. 556-566.

(51) KAnNeR, L., «To what Extent is Early Infantile Autism Determined by Constitutional Inadequacies?», en KanNer, L., Childhood Psychosis: Initial Studies and New Insights, Washington, V. H. Winston and Sons, 1973, pp. 69-75.

(52) Hobson, R. P., «Origins of the Personal Relation and the Estrange Case of Autism», Association for Child Psychology and Psychiatry, 1983a.

(53) Hobson, R. P., «The Autistic Child's Recognition of Age-Related Features of People, Animals and Things», British Journal of Developmental Psychology, 1983b, 1, pp. 343-352.

(54) Hobson, R. P., «Early Childhood Autism and the Question of Egocentrism», Journal of Autism and Developmental Disorders, 1984, 14, pp. 85-104.

(55) Hobson, R. P., «Beyond Cognition. A Theory of Autism», en Dawson, G. (ed.), Autism, Nature, Diagnosis, and Treatment, Nueva York, The Guilford Press, 1989.

(56) RiviÈRE, A., IDEA: inventario de espectro autista, Buenos Aires, Fundec, 2002.

(57) Hobson, R. P., El autismo y el desarrollo de la mente, Madrid, Alianza, 1995.

(58) Leslie, A. M.; Frith, U., «Prospects for Cognitive Neuropsychology of Autism, Hobson's Choice», Psychological Review, 1989, 97, pp. 122-131.

(59) Leslie, A. M.; HAPPE, P., «Autism and Ostensive Communication: The Relevance of Metarepresentation», Development and Psychopathology, 1989.

(60) Baron-Cohen, S.; Howlin, P., «The Theory of Mind Deficit in Autism: some Questions for Teaching and Diagnosis», en Baron-Cohen, S.; Tager-Flusberg, H.; Cohen, D. (eds.), Understanding other Minds. Perspectives from Autism, Nueva York, Oxford University Press, pp. 466-480, 1993.

(61) Dawson, G.; Lewy, A., «Arousal, Attention, and the Socialemotional Impairments of Individuals with Autism», en Dawson, G. (ed.), Autism, Nature, Diagnosis, and Treatment, NuevaYork, The Guilford Press, 1989.

(62) Newson, C. D.; Rincover, A., «Behavioral Assessment of Autistic Children», en E. Mash, J.; Terdal, L. G. (eds.), Behavioral Assessment of Childhood Disorders, Nueva York, Guilford Press, 1982.

(63) RIVIÈRE, A., y otros, Evaluación y alteraciones de las funciones psicológicas en el autismo, Madrid, Ministerio de Educación y Ciencia (Centro de Investigación y Documentación Educativa), 1988. 
(64) Lord, C.; Rutter, M.; Le Couteur, A., Autism Diagnostic Interview-Revised (ADI-R): A Revised Version of Diagnostic Interview for Caregivers of Individuals with Possible Pervasive Developmental Disorder, Journal of Autism and Developmental Disorders, 1994, 24, pp. 659-685.

(65) SCHOPLER, E., «Modificación de la participación parental en el tratamiento comportamental», en RutTer, M.; Schopler, E. (coords.), Autismo. Reevaluación de los conceptos y el tratamiento, Madrid, Alhambra, 1984, pp. 393-401.

(66) Palomo, R., y otros, «Evaluación y diagnóstico en trastornos del espectro autista: el modelo IRIDIA», en VALDEZ, D. (comp.), Evaluar e intervenir en autismo, Madrid, A. Machado Libros, 2005, pp. 45-95.

(67) CANAL, R., «El autismo hoy en España», entrevista al doctor Ángel Rivière, Siglo Cero, 1993, 149, 24, pp. 41-44.

* Francisco Balbuena Rivera, psicólogo, departamento de Psicología, Universidad de Huelva. Correspondencia: Francisco Balbuena Rivera, balbuena@uhu.es. Avda. Luis Montoto, 130, A-1-1. 41005 Sevilla.

** Fecha de recepción: 4-I-2007. 